\title{
Cardiopatias congênitas e sua associação com o uso de antidepressivos na gestação: uma revisão sistemática
}

\author{
Congenital heart diseases and their association with the use of antidepressive agents in \\ pregnancy: a systematic review
}

Cardiopatía congénita y su asociación con el uso de antidepresivos en el embarazo: una revisión sistemática

Natália Rodrigues Gomes de Assis ${ }^{1 *}$, Bárbara Nogueira Roberti ${ }^{1}$, Lorena de Souza Carvalho ${ }^{1}$, Lucas Palhares Barreto Mendes ${ }^{1}$, Luis Gustavo Fernandes Dias ${ }^{1}$, Luiza Marques ${ }^{1}$, Maria Luisa Lara Lanza Stabile$^{1}$, Paola Falcão Moreira Nogueira1', Eduardo Augusto dos Santos Moreira Silva1.

\section{RESUMO}

Objetivo: Analisar a literatura acerca da relação entre os antidepressivos mais utilizados por gestantes e o aumento da incidência de cardiopatias congênitas no recém-nascido (RN). Métodos: Por meio dos descritores "Cardiopatias Congênitas" e "Antidepressivos", realizou-se uma busca nas bases de dados MEDLINE/PubMed, SciELO, LILACS e CAPES. Como critérios de inclusão, foram aceitos artigos em inglês, português e espanhol, limitados aos últimos 5 anos (2015-2020) e que abordassem o uso de Inibidores Seletivos da Recaptação de Serotonina (ISRS) ou Inibidores Seletivos da Recaptação de Serotonina e Noradrenalina (IRSN) em qualquer momento da gestação. Foram excluídos os artigos sem resumo ou sem texto completo, as duplicidades, as cartas ao editor e os referentes à cardiopatias em adultos e animais. Resultados: Inicialmente 29 artigos foram encontrados, destes, 6 atenderam os critérios e analisaram a influência de IRSN ou de ISRS nas malformações cardíacas no RN. Considerações finais: Com base nos artigos recuperados, não existe um consenso sobre a associação do uso de ISRS ou de IRSN durante a gestação e o desenvolvimento de cardiopatias congênitas.

Palavras-chave: Cardiopatias congênitas, Antidepressivos, Gravidez.

\section{ABSTRACT}

Objective: To analyze the literature on the relationship between the antidepressive agents most used by pregnant women and the increased incidence of congenital heart disease in the newborn (NB). Methods: Using the descriptors "Heart Defects, Congenital" and "Antidepressive Agents", a search was performed in the databases MEDLINE/ PubMed, SciELO, LILACS and CAPES. As inclusion criteria, articles in English, Portuguese and Spanish were accepted, limited to the last 5 years (2015-2020) and that addressed the use of Selective Serotonin Reuptake Inhibitors (SSRIs) or Selective Serotonin and Noradrenaline Reuptake Inhibitors (SNRI) at any time during pregnancy. Articles without abstract or full text, duplicated, letters to the editor and those referring to heart diseases in adults and animals were excluded. Results: Initially, 29 articles were found, of these, 6 attended the criteria and discussed the influence of SNRI or SSRIs on cardiac malformations in newborns. Final considerations: Based on the retrieved articles, there isn't consensus on the association between the use of SSRIs or SNRI during pregnancy and the development of congenital heart diseases.

Key words: Heart congenital, Antidepressive agents, Pregnancy.

\section{RESUMEN}

Objetivo: Analizar la literatura sobre la relación entre los antidepresivos más utilizados por mujeres embarazadas y la subida de la incidencia de las cardiopatías congénitas en el recién nacido (RN). Métodos: Por medio de los descriptores "Cardiopatías Congénitas" y "Antidepresivos", se ha realizado una búsqueda en las bases de datos MEDLINE/PubMed, SciELO, LILACS y CAPES. Como criterios de inclusión, han sido aceptados artículos en inglés, portugués y español, limitados a los últimos 05 años (2015-2020) y que tuvieran como tema el uso de Inhibidores Selectivos de la Re-captación de Serotonina (ISRS) o Inhibidores Selectivos de la Re-captación de Serotonina y Noradrenalina (IRSN) en cualquier momento del embarazo. Han sido

${ }^{1}$ Pontifícia Universidade Católica de Minas Gerais (PUC-MG), Betim - MG.

*E-mail: nataliadiassis@hotmail.com

SUBMETIDO EM: 7/2020

ACEITO EM: 8/2020

PUBLICADO EM: 10/2020

REAS/EJCH | Vol.12(10) | e4715 | DOI: https://doi.org/10.25248/reas.e4715.2020 
excluidos los artículos sin resumen o sin texto completo, las duplicidades, las cartas al editor y los referentes a las cardiopatías en adultos y animales. Resultados: Inicialmente 29 artículos han sido encontrados, de éstos, 06 han atendido a los criterios y analizaban la influencia de IRSN o de ISRS en las malas formaciones cardiacas en el RN. Consideraciones finales: Con base en los artículos recuperados, no hay un consenso sobre la vinculación del uso de ISRS o de IRSN durante el embarazo al desarrollo de cardiopatías congénitas.

Palabras clave: Cardiopatías congénitas, Antidepresivos, Embarazo.

\section{INTRODUÇÃO}

Os cenários mundial e nacional têm apresentado a depressão como uma das doenças de maior incidência na atualidade, sendo o risco de desenvolvimento desta maior entre mulheres (ORGANIZAÇÃO MUNDIAL DE SAÚDE, 2018); com manifestação frequente durante a gestação. Nesse contexto, destacam-se as mudanças físicas, fisiológicas, sociais e psicológicas que são próprias da gravidez e que tornam as gestantes um grupo vulnerável ao desenvolvimento de transtornos depressivos.

Assim, os fatores de risco mais relevantes são aqueles relacionados às emoções e aos sentimentos da gestante, como infelicidade ou tristeza com a gravidez, alterações hormonais importantes, além de estressores psicossociais, como a preocupação com a gestação e a adaptação a novas situações de vida (SADOCK BJ, 2017).

Dentre estes, pode-se citar, ainda, o uso de substâncias psicoativas, a exemplo o álcool e o tabaco, além da ausência de apoio social e da própria família (SILVA MMJ, et al., 2020). Ademais, questões relacionadas a condições obstétricas também podem estar presentes, como a ocorrência de doenças maternas durante a gravidez, complicações gestacionais e história pregressa de aborto ou de natimorto.

A partir do diagnóstico de um quadro depressivo em uma gestante, cabe ao médico responsável realizar a escolha do antidepressivo mais adequado para o subtipo de depressão daquela paciente, tendo em mente a seleção do medicamento que atribua menor risco ao feto e à mãe. Atualmente, destaca-se que a classe de antidepressivos mais utilizada no tratamento para jovens e adultos é a dos Inibidores Seletivos de Recaptação da Serotonina (ISRS), sendo mais comuns para as gestantes, a Fluoxetina e a Sertralina (MORENO RA e CORDÁS TA, 2018). Destaca-se, ainda, que a ação seletiva desta medicação garante um perfil mais tolerável de efeitos colaterais (RIBEIRO AG, et al., 2014).

Outra classe medicamentosa muito utilizada atualmente é a dos Inibidores de Recaptação de Serotonina e Noradrenalina (IRSN), como a Venlafaxina e a Duloxetina. Conhecidos como duais, esses medicamentos apresentam benefícios não só para quadros depressivos, mas também para quadros de ansiedade associados. Essa classe costuma ser, portanto, prescrita com certa frequência para gestantes, visto que muitas desenvolvem ansiedade devido à condição de espera na qual se encontram (BRUNTON LL, 2012).

Entretanto, assim como qualquer medicamento, os antidepressivos não estão isentos de efeitos colaterais e de riscos. Dessa forma, algumas literaturas apresentam os potenciais efeitos teratogênicos do uso materno desses medicamentos no desenvolvimento de anomalias fetais, como as cardiopatias congênitas do recémnascido (RN).

A doença cardíaca congênita é qualquer alteração na anatomia do coração e seus vasos sanguíneos que ocorre nas primeiras 8 semanas de gestação (SOARES AM, 2018). As manifestações clínicas dela ocorrem principalmente nos primeiros meses de idade, porém, a doença pode ser reconhecida em qualquer momento da vida. A evolução natural desta cursa com mortalidade extremamente alta, sendo que cerca de $20 \%$ das crianças morrem nos primeiros 12 meses de vida (SOCIEDADE BRASILEIRA DE PEDIATRIA, 2017).

As cardiopatias congênitas podem ser classificadas em cardiopatia de hipofluxo pulmonar, cardiopatia de hiperfluxo pulmonar e cardiopatia de normofluxo pulmonar, sendo que a suspeita clínica no período neonatal pode ser sugerida pela presença de quatro achados principais: sopro cardíaco, cianose, taquipneia e arritmia cardíaca (AMARAL F, et al., 2002).

Nesse contexto, ressalta-se o fato de o sopro cardíaco ser um achado comum no recém-nascido (RN), que deve ser classificado prontamente como inocente ou patológico, avaliando a probabilidade de estar ou 
não relacionado a uma cardiopatia. Assim, sopros patológicos comumente são caracterizados como rudes, normalmente sistólicos e usualmente localizados (AMARAL F, et al., 2002). Além disso, as alterações mais encontradas relacionadas à origem dos sopros são a comunicação interventricular, a comunicação interatrial e a permanência do canal atrial, potencialmente capazes de originar sons incomuns que, muitas vezes, geram a primeira suspeita de uma cardiopatia congênita (TEIXEIRA BC e TALIARI JDS, 2018).

Além do sopro, outra manifestação clínica muito frequente nas crianças é a cianose, encontrada principalmente nos casos de cardiopatias congênitas com transposição simples das grandes artérias e na Tetralogia de Fallot (SOCIEDADE BRASILEIRA DE PEDIATRIA, 2017). No caso da transposição, as grandes artérias encontram-se invertidas, e o sangue com pouco oxigênio transcorre para a circulação sistêmica, enquanto o sangue mais oxigenado é direcionado aos pulmões.

Consequentemente, essa situação cursa com baixa oxigenação sistêmica e dificuldade respiratória, o que aumenta o esforço cardíaco, podendo ter a cianose como apresentação clínica associada (FAZOLIN MAFG, et al., 2016). Já a Tetralogia de Fallot é uma cardiopatia composta por quatro malformações: comunicação do septo interventricular, a qual ocorre quando existe um orifício entre os dois ventrículos; dextroposição da aorta, indicativa de um desalinhamento da aorta para a direita ao sair do coração; obstrução do fluxo sanguíneo do ventrículo direito e hipertrofia ventricular direita (NASCIMENTO MNB, et al., 2017).

Deve-se atentar também à possibilidade de cardiopatia congênita sempre que um RN se apresentar com quadro de taquipneia ou de dispneia, sendo que a maioria dos pacientes com taquipneia secundária à cardiopatia costuma apresentar também alterações semiológicas específicas, como sopro cardíaco, alterações nas bulhas cardíacas, presença de ruídos acessórios e anormalidades nos pulsos arteriais. Assim, as principais causas cardíacas de taquidispneia neonatal de origem congênita são a persistência do canal arterial no RN prematuro e a estenose valvar aórtica grave. Já os distúrbios de ritmo cardíaco podem ser detectados acidentalmente, quando da avaliação neonatal rotineira ou, mais raramente, em situação de encaminhamento do RN para a urgência (AMARAL F, et al., 2002).

Assim, no contexto atual em que a depressão é um dos grandes problemas e uma das principais doenças da população mundial, faz-se necessário estudar todas as suas facetas. Dentre elas, é importante avaliar a possível associação entre o tratamento medicamentoso dos transtornos depressivos durante a gestação e o risco de desenvolvimento de cardiopatias congênitas no feto, condição esta com alta morbi-mortalidade ao RN. Portanto, esse trabalho objetiva, por meio de uma revisão sistemática da literatura, fazer uma análise de estudos que apontam se alguns dos antidepressivos muito utilizados atualmente, os ISRS e os IRSN estão ou não relacionados com um aumento da incidência de cardiopatias congênitas no RN.

\section{MÉTODOS}

Este estudo trata-se de uma revisão sistemática da literatura acerca da relação entre o uso de antidepressivos durante a gestação e sua associação com um risco maior dos fetos desenvolverem malformações congênitas, em especial, cardiopatias congênitas.

Para a construção da pergunta de pesquisa e da busca bibliográfica de evidência utilizou-se a estratégia PICO, com os seguintes termos para o acrônimo (P) Gestantes; (I) Uso de antidepressivos; (C) Não utilizar antidepressivos; (O) Aumento do risco de cardiopatia congênita em recém nascidos, tendo como questão clínica: há evidências de que o uso de antidepressivos durante a gestação aumenta o risco de cardiopatia congênita nos recém nascidos? Para tanto, foram utilizadas as seguintes bases de dados: Medical Literature Analysis and Retrieval System Online (MEDLINE), Scientific Electronic Library Online (SciELO), Literatura Latino-Americana e do Caribe em Ciências da Saúde (LILACS), Coordenação de Aperfeiçoamento de Pessoal de Nível Superior (CAPES) e PubMed.

Os descritores utilizados para a busca dos artigos foram levantados pelo DeCS da BVS (Biblioteca Virtual de Saúde), sendo eles: "antidepressive agents" e "heart defects, congenital". Para realizar a combinação dos descritores, foi utilizado o operador booleano "AND". Somente textos com tais descritores foram selecionados para avaliação individual dos autores. Essa revisão é composta por 6 etapas: (1) escolha da temática; (2) elaboração da questão norteadora através da estratégia PICO; (3) busca de evidências por meio da escolha 
dos descritores e das bases de dados a serem utilizadas; (4) determinação dos critérios de seleção dos artigos que seriam pertinentes ou não para a pesquisa; (5) análise dos artigos; e (6) apresentação de discussão e resultados.

Os critérios de inclusão definidos para a seleção dos artigos foram: artigos publicados nos últimos 5 anos (2015-2020) em inglês, português e espanhol; que tratassem de gestantes que fizeram uso de Inibidores Seletivos da Recaptação de Serotonina (ISRS) ou Inibidores de Recaptação de Serotonina e Noradrenalina (IRSN) em qualquer momento da gestação; que fizessem menção no título ou resumo à associação entre o uso de antidepressivos seletivos ou duais durante a gestação e o desenvolvimento de cardiopatias congênitas em recém nascidos.

Os critérios de exclusão foram: artigos sem resumo e que não apresentavam texto completo, estudos em que o medicamento em uso era diferente de ISRS ou de IRSN; artigos duplicados; publicações que se tratavam de carta ao editor e artigos que apresentavam estudos sobre cardiopatia congênita em adultos e em animais.

Na combinação dos descritores "antidepressive agents" AND "heart defects, congenital", e após a aplicação dos filtros "ano de publicação", abrangendo artigos publicados entre 2015 a 2020, e "idioma", incluindo aqueles escritos em inglês, português e espanhol, foram encontrados treze (13) artigos na PubMed, nove (9) no MEDLINE e sete (7) no CAPES, totalizando vinte e nove (29). Não houve correspondências na SCiELO e na LILACS.

Após essa primeira seleção, os critérios de exclusão foram aplicados primeiramente a partir da leitura dos títulos e dos resumos. Descartou-se, então, cartas ao editor, artigos duplicados, incompletos ou sem resumo, que não apresentavam relação com o objeto de estudo, ou que não foram realizados com humanos, sendo selecionados, portanto, cinco (5) da PubMed e três (3) da MEDLINE. Após a leitura completa dos artigos, excluiu-se um (1) artigo da Pubmed que não correspondia ao objetivo do nosso estudo e um (1) artigo da MEDLINE que se tratava de um comentário dos autores. Com isso, após aplicação dos critérios mencionados, foram escolhidas as publicações consideradas relevantes para o trabalho, sendo selecionados seis (6) artigos para integrar esta revisão sistematizada. A Figura 1 ilustra as etapas utilizadas para seleção dos artigos em um fluxograma.

Figura 1 - Fluxograma com as etapas de seleção dos artigos. Belo Horizonte, MG, Brasil, 2020.
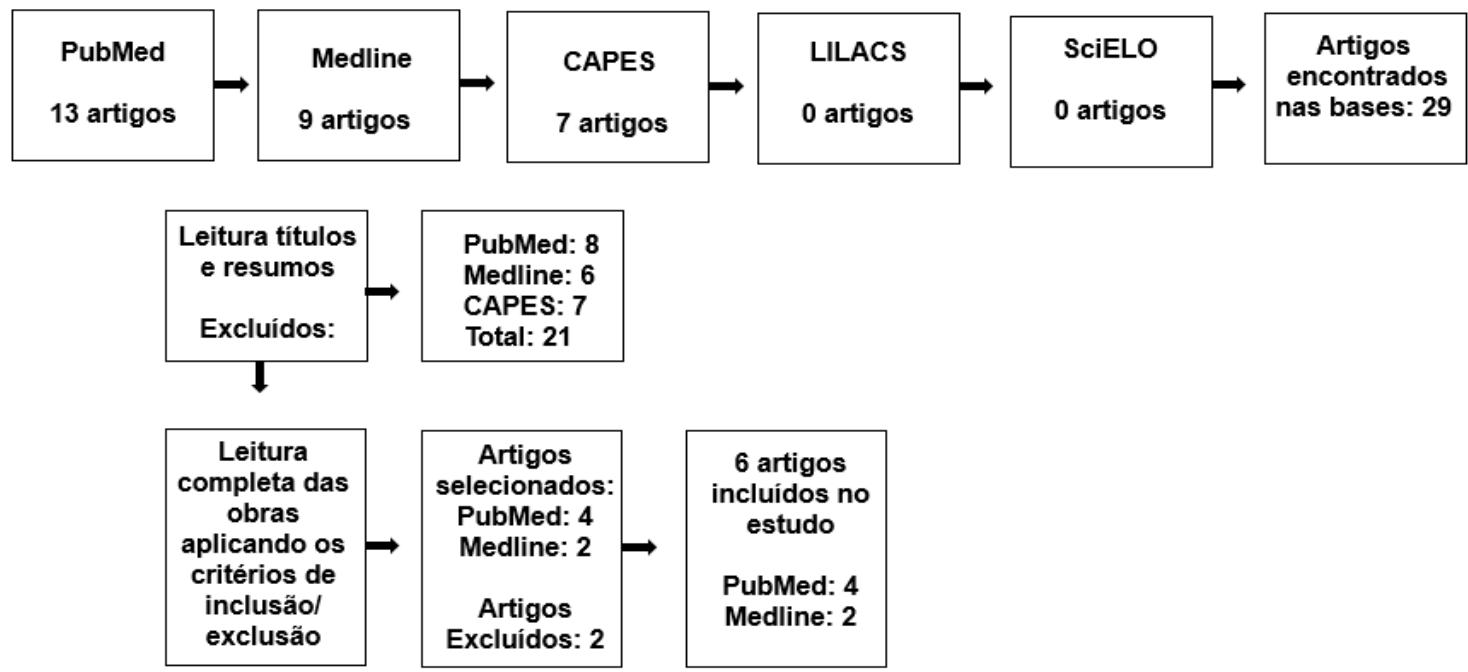

Fonte: Assis NRG, et al., 2020.

\section{RESULTADOS}

Após a seleção final, os artigos foram organizados conforme autor, ano de publicação, desenho do estudo, objetivo e conclusão. Todos os seis artigos foram publicados em inglês. Em relação ao ano de publicação, dois foram publicados em 2015, três em 2016, e apenas um em 2020. O Quadro 1 exibe os artigos selecionados e os critérios abordados conforme previamente citado. 
Quadro 1 - Artigos recuperados na revisão sistemática.

\begin{tabular}{|c|c|c|c|c|}
\hline Autor & $\begin{array}{l}\text { Ano de } \\
\text { Publicação }\end{array}$ & $\begin{array}{l}\text { Desenho } \\
\text { de Estudo }\end{array}$ & Objetivo do Estudo & Conclusão \\
\hline $\begin{array}{l}\text { BÉRARD A, } \\
\text { et al. (2016) }\end{array}$ & 2016 & $\begin{array}{l}\text { Revisão } \\
\text { Sistemática } \\
\text { com } \\
\text { Metanálise }\end{array}$ & $\begin{array}{l}\text { Realizar meta-análise sobre o risco de malformações } \\
\text { cardíacas associadas à exposição gestacional à } \\
\text { paroxetina. }\end{array}$ & $\begin{array}{l}\text { Defende-se que a paroxetina tenha mais riscos do que benefícios se } \\
\text { usada durante a organogênese, sendo, portanto, discutíveis seus } \\
\text { benefícios. Dessa forma, qualquer aumento no risco é tido como } \\
\text { significativo. }\end{array}$ \\
\hline $\begin{array}{l}\text { DAUD A, et } \\
\text { al. }(2016)\end{array}$ & 2016 & $\begin{array}{l}\text { Revisão de } \\
\text { Literatura }\end{array}$ & $\begin{array}{l}\text { Explorar se preditores farmacogenéticos envolvidos na } \\
\text { farmacocinética e mecanismo de ação dos ISRS têm } \\
\text { relação com a discrepância nas estimativas de risco } \\
\text { de desenvolvimento de cardiopatias congênitas. }\end{array}$ & 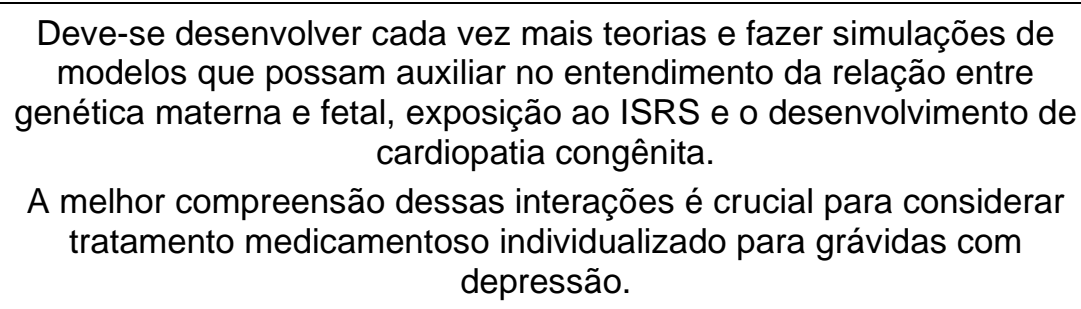 \\
\hline $\begin{array}{l}\text { HUYBRECH } \\
\text { TS K, et al. } \\
\text { (2020) }\end{array}$ & 2020 & $\begin{array}{l}\text { Estudo de } \\
\text { Coorte } \\
\text { retrospectivo }\end{array}$ & $\begin{array}{l}\text { Avaliar o risco de resultados maternos e infantis } \\
\text { adversos após a exposição intrauterina à duloxetina. }\end{array}$ & $\begin{array}{l}\text { Considerando as evidências existentes, parece improvável que a } \\
\text { duloxetina apresente efeitos teratogênicos significativos. Entretanto, } \\
\text { pode estar associada a um aumento do risco de hemorragia pós- } \\
\text { parto e discreto risco de malformações cardíacas congênitas. Apesar } \\
\text { de incomuns, os benefícios do uso durante a gestação devem ser } \\
\text { ponderados até que se tenham mais estudos. }\end{array}$ \\
\hline $\begin{array}{l}\text { JORDAN S, } \\
\text { et al. (2016) }\end{array}$ & 2016 & $\begin{array}{l}\text { Estudo de } \\
\text { Coorte } \\
\text { Prospectivo }\end{array}$ & $\begin{array}{c}\text { Investigar o suposto efeito teratogênico na prescrição } \\
\text { dos ISRS durante } 91 \text { dias antes e depois da data da } \\
\text { última menstruação (DUM). }\end{array}$ & $\begin{array}{l}\text { O risco absoluto de teratogenia associado aos ISRS, é casualmente } \\
\text { pequeno. Entretanto, devido à prevalência elevada do uso de ISRS } \\
\text { aumenta sua importância em saúde pública, justificando } \\
\text { modificações nos cuidados pré-concepção. }\end{array}$ \\
\hline $\begin{array}{l}\text { SHEALY K } \\
(2015)\end{array}$ & 2015 & $\begin{array}{l}\text { Revisão } \\
\text { narrativa de } \\
\text { literatura }\end{array}$ & $\begin{array}{l}\text { Questionar os riscos verdadeiros de uso de } \\
\text { antidepressivos, principalmente dos ISRS no primeiro } \\
\text { trimestre de gestação. }\end{array}$ & $\begin{array}{l}\text { Pacientes e médicos devem discutir os riscos e os benefícios do uso } \\
\text { da medicação, principalmente no período pré concepção, visto que o } \\
\text { tratamento da depressão deve ser individualizado. }\end{array}$ \\
\hline $\begin{array}{l}\text { WANG S, et } \\
\text { al. (2015) }\end{array}$ & 2015 & $\begin{array}{l}\text { Revisão } \\
\text { sistemática } \\
\text { com } \\
\text { Metanálise } \\
\text { com artigos de } \\
\text { coorte }\end{array}$ & $\begin{array}{l}\text { Avaliar a associação entre ISRS no primeiro trimestre } \\
\text { de gestação, e o risco de desenvolvimento de defeitos } \\
\text { cardíacos congênitos. }\end{array}$ & $\begin{array}{l}\text { Os ISRS durante o primeiro trimestre em mulheres grávidas não } \\
\text { foram associados a riscos aumentados para defeitos cardíacos no } \\
\text { recém-nascido. }\end{array}$ \\
\hline
\end{tabular}

Fonte: Assis NRG, et al., 2020.

REAS/EJCH | Vol.12(10) | e4715 | DOI: https://doi.org/10.25248/reas.e4715.2020 Página 5 de 8 


\section{DISCUSSÃO}

Os transtornos depressivos são altamente prevalentes no contexto atual, tendo o pico de manifestação entre os 20 e os 50 anos (SADOCK BJ, et al., 2017). Nesse cenário, mulheres em idade fértil e que, portanto, podem potencialmente engravidar, estão fortemente suscetíveis à depressão e, consequentemente, ao tratamento medicamentoso. Somado a isso, segundo Pereira PK e Lovisi GM (2008); o período gravídicopuerperal é a fase de maior prevalência de transtornos mentais na mulher, visto que nesse período a gestante passa por uma série de alterações psíquicas, sendo que o modo com que a mesma lidará com essa situação dependerá de fatores orgânicos, familiares, conjugais, sociais e da própria personalidade da gestante.

Segundo Wang S, et al. (2015), a depressão afeta cerca de 15\% das gestantes e quadros não tratados podem estar associados à aborto, à prematuridade e ao muito baixo peso fetal. Concordante com esse estudo, Shealy K (2015) afirma que devem ser considerados os potenciais riscos da descontinuação das medicações, como a maior possibilidade de recaída da doença, automutilação, depressão pós-parto, má nutrição materna e ganho de peso inadequado ao decorrer da gestação. De acordo com esses autores, portanto, faz-se necessário o tratamento adequado dos quadros depressivos na gestação.

Os transtornos depressivos, assim como outros transtornos psiquiátricos, têm o tratamento farmacológico baseado em antidepressivos. Atualmente, as medicações mais utilizadas no tratamento dessas condições são, preferencialmente, os chamados Inibidores Seletivos de Recaptação de Serotonina (ISRS) e os Inibidores de Recaptação de Serotonina e Noradrenalina (IRSN), visto que ambos apresentam resultados satisfatórios e provocam efeitos colaterais mais brandos quando comparados às outras classes (SHEALY K, 2015).

Os antidepressivos são classificados pelo Food and Drug Administration (FDA) conforme o risco associado ao seu uso durante a gravidez, sendo que a maioria destes são classificados como ranque $\mathrm{C}$ ou $\mathrm{D}$. Isso indica que para este há alguma evidência de risco fetal, e que para aquele não há estudos bem controlados em humanos e/ou modelos animais que demonstrem potencial prejuízo para o feto (SHEALY K, 2015).

Nesse contexto, acredita-se se que alguns antidepressivos possam estar relacionados à certo grau de teratogenicidade, visto que a passagem das excretas metabólicas dos ISRS e IRSN ocorrem pelo saco vitelínico, desde o estágio inicial da gestação até a formação da placenta, no primeiro trimestre. Os metabólitos chegam ao cordão umbilical e sua presença no líquido amniótico prolonga a exposição do feto a essas substâncias (JORDAN S, et al., 2016). Dessa forma, o desenvolvimento de efeitos adversos devido à medicação está relacionado ao tempo e à duração da exposição (SHEALY K, 2015).

Corroborando o estudo de Shealy K, et al. (2015), por meio de uma análise dos registros de três países europeus, Jordan S, et al. (2016) encontraram associações positivas, mas não significativas, envolvendo todos os ISRS e as doenças cardíacas congênitas (DCC). Em especial, a paroxetina foi relacionada à ocorrência de defeitos cardíacos; ademais houve maior prevalência de DCC grave em casos de uso de doses mais elevadas desses medicamentos e menor prevalência em RN cujas mães interromperam o tratamento.

Outro estudo, realizado por Bérard A, et al. (2016), também mostrou aumento no risco de complicações cardíacas congênitas associado ao uso de paroxetina, em que a exposição durante o primeiro trimestre de gestação foi relacionada a um risco duas vezes maior de defeitos no septo atrial e de obstrução da via de saída do ventrículo direito, quando comparado à não exposição à medicação durante a gravidez. É importante lembrar que o aumento desse risco é diretamente influenciado pelo tempo de diagnóstico da alteração cardíaca, sobretudo em defeitos do septo atrial, que podem não ser detectados através da ausculta cardíaca no primeiro ano de vida da criança (BÉRARD A, et al., 2016).

Desse modo, é válido ressaltar que os malefícios do uso da paroxetina ao longo da gestação são discutidos desde 2005, quando a FDA alterou a categoria desse ISRS de C para D. Vê-se então que os resultados expostos por Bérard A, et al. (2016) estão de acordo com o apresentado pela revisão de Daud AN, et al. (2016). Nesta revisão é ressaltada a importância do conhecimento sobre a farmacocinética dos ISRS e dos IRSN para melhor compreensão da interação da exposição fetal a esses fármacos e o risco de desenvolvimento dos defeitos cardíacos congênitos. 
Assim, esse risco pode estar associado à concentração materna e fetal de serotonina, fator esse que pode ser modificado por alterações metabólicas e genéticas, como a expressão e a função de enzimas, de proteínas transportadoras e de receptores serotoninérgicos (DAUD AN, et al., 2016).

Durante a embriogênese, o feto tem suprimentos de serotonina (5-HT) oriundos do sangue materno, por meio da expressão dos transportadores de serotonina na placenta. Em mães com transtornos depressivos, há uma disfunção serotoninérgica no Sistema Nervoso Central (SNC), que pode repercutir em alterações no feto. Ao passo que, ao usar os ISRS ou os IRSN, esses podem inibir os transportadores serotoninérgicos nas células cardíacas fetais, o que, consequentemente, diminui o transporte de $5-\mathrm{HT}$, causando um distúrbio na organogênese cardíaca, uma vez que, a serotonina atua de maneira indireta nesse processo e é um dos elementos envolvidos na lateralidade das células do coração.

Portanto, alterações nos níveis de 5-HT afetam a morfogênese cardíaca e resultam em defeitos do coração como transposição das grandes artérias e ventrículo direito de dupla saída. Ainda segundo os autores, apesar do risco absoluto ser considerado baixo, um estudo anterior relatou a associação de defeitos de obstrução da via de saída do ventrículo direito ao uso de paroxetina e de fluoxetina durante a gestação (DAUD AN, et al., 2016).

Wang S, et al. (2015), realizaram uma metanálise na qual foram analisados quatro coortes, dos quais três concluíram não haver associação entre o uso materno de ISRS e o aumento das anomalias cardíacas congênitas. Desse modo, também chegaram à conclusão de que parece não haver correlação entre o uso de ISRS e o aumento do risco de defeitos cardíacos em recém-nascidos.

Ademais, foi publicado um estudo de coorte avaliando o risco para a mãe e para o feto após a exposição à duloxetina, um antidepressivo dual, durante a gestação. O estudo comparou a utilização da medicação com quatro grupos controle, e a partir disso foi sugerido que é improvável que a duloxetina seja um teratogênico importante, visto que não se encontrou diferenças significativas entre os grupos, embora tenha sido identificado um pequeno aumento no risco de DCC e de hemorragia pós parto (HUYBRECHTS K, et al., 2020).

Diversos estudos foram publicados relacionando o uso de antidepressivos à malformações cardíacas congênitas. No entanto, apresentaram resultados inconclusivos ou conflitantes. Haja vista que, esses trabalhos contam com vários fatores de confusão e vieses relacionados à critérios de exposição, população e resultados. Esse contexto é somado a dificuldade da obtenção de uma amostra grande o suficiente para confirmar a associação em questão, visto que as anomalias cardíacas congênitas acometem oito a cada mil bebês nascidos vivos e os possíveis riscos dos ISRS e dos IRSN despertam preocupações nas pacientes que muitas vezes optam por não realizar o tratamento farmacológico e assim a exposição dos fetos a medicação também é pouco frequente (DAUD AN, et al., 2016).

Jordan S, et al. (2016) corrobora o estudo de Daud AN, et al. (2016), ao manifestar os obstáculos estatísticos e éticos da realização de estudos randomizados que possuem como desfecho o aumento do risco de cardiopatias congênitas. Todavia os autores afirmam que os resultados dos estudos observacionais e dos ensaios realizados até o momento, devem ser analisados e cuidadosamente implicados no tratamento de caráter individual da depressão durante o período gestacional.

\section{CONSIDERAÇÕES FINAIS}

Com base nos artigos encontrados, não existe ainda um consenso sobre a associação entre o uso de ISRS ou de IRSN durante a gestação e o desenvolvimento de cardiopatias congênitas. Tal discordância está relacionada, sobretudo, às variações nas metodologias e nas análises dos resultados dos estudos. Nesse cenário, enquanto a literatura não oferece consistência e a farmacogenética não é amplamente empregada, a melhor prática envolve a avaliação dos benefícios do tratamento da depressão e dos fatores de risco individuais da paciente. Esta revisão sistemática, tem como limitação a pequena amostra de trabalhos recuperados, o que evidencia a escassez de estudos, nos últimos cinco anos, acerca da abordagem terapêutica da depressão em gestantes e o aumento do risco de anomalias congênitas, com foco particular nas malformações cardíacas. Assim, recomendamos a realização de mais pesquisas, com alto grau de evidência, que envolvam os temas abordados. 


\section{REFERÊNCIAS}

1. AMARAL F, et al. Quando suspeitar de cardiopatia congênita no recém-nascido. Medicina. Ribeirão Preto Online, 2002; 35(2): 192-197.

2. BÉRARD A, et al. The risk of major cardiac malformations associated with paroxetine use during the first trimester of pregnancy: a systematic review and meta-analysis. Br J Clin Pharmacol, 2016; 81(4): 589-604.

3. BRUNTON LL, et al. Goodman \& Gilman: As Bases Farmacológicas da Terapêutica. 13 ${ }^{\text {a }}$ ed. Rio de Janeiro: AMGH, 2018; 1760p.

4. DAUD AN, et al. The Risk of Congenital Heart Anomalies Following Prenatal Exposure to Serotonin Reuptake Inhibitors-Is Pharmacogenetics the Key?. Int J Mol Sci, 2016; 17(8): 1333.

5. FAZOLIN MAFG, et al. Treinamento de força para indivíduos com transposição das grandes artérias: uma revisão. Revista Brasileira de Prescrição e Fisiologia do Exercício, 2016; 10(61): 684-691.

6. HUYBRECHTS K, et al. Maternal and fetal outcomes following exposure to duloxetine in pregnancy: cohort study. BMJ, 2020; 368:237.

7. JORDAN S, et al. Selective Serotonin Reuptake Inhibitor (SSRI) Antidepressants in Pregnancy and Congenital Anomalies: Analysis of Linked Databases in Wales, Norway and Funen, Denmark. Plos One, 2016; 11(12): $122-165$.

8. MORENO RA, CORDÁS TA. Condutas em psiquiatria: consulta rápida. $2^{2}$ ed. Porto Alegre: Artmed, 2018; 456p.

9. NASCIMENTO MNB, et al. Assistência de enfermagem em crianças com tetralogia de Fallot. Congresso internacional de enfermagem anais, 2017; 1(1).

10. ORGANIZAÇÃO MUNDIAL DE SAÚDE, 2018. Folha informativa - Depressão. Brasília: Organização Panamericana de Saúde.

11. PEREIRA PK, LOVISI GM. Prevalência de depressão gestacional e fatores associados. Rev. Psiquiatr. Clin., 2008; 35(4): 144-153.

12. RIBEIRO AG, et al. Antidepressivos: uso, adesão e conhecimento entre estudantes de medicina. Ciência \& Saúde Coletiva, 2014; 19(6): 1825-1833.

13. SADOCK BJ, et al. Compêndio de psiquiatria: ciência do comportamento e psiquiatria clínica. $11^{a}$ ed. Porto Alegre: Artmed, 2017; 1490p.

14. SHEALY K. Are antidepressants safe in the first trimester of pregnancy?. Jornal da Academia Americana de APs, 2015; 28(4): 16-17.

15. SILVA MMJ, et al. Depressão na gravidez. Smad Revista Eletrônica Saúde Mental Álcool e Drogas (edição em português), 2020; 16(1): 1-12.

16. SOARES, AM. Mortalidade por doenças cardíacas congênitas críticas e fatores de risco associados em recémnascidos. Um estudo de coorte. Arq. Bras. Cardiol., 2018; 111(5): 674-675.

17. SOCIEDADE BRASILEIRA DE PEDIATRIA. Tratado de Pediatria. 4aa ed. São Paulo: Manole, 2017; $2471 \mathrm{p}$.

18. TEIXEIRA BC, TALIARI JDS. Sopro cardíaco em neonatos portadores de cardiopatia congênita. Revista Funec Científica - Enfermagem, 2018; 2(3): 65-72.

19. WANG S, et al. Selective Serotonin Reuptake Inhibitors (SSRIs) and the Risk of Congenital Heart Defects: A MetaAnalysis of Prospective Cohort Studies. J Am Heart Assoc, 2015; 4(5): 1-7. 